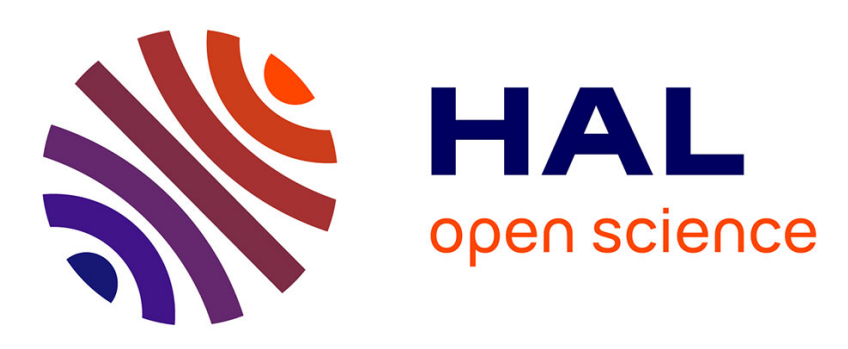

\title{
Prolonger l'inaction environnementale dans un monde familier : la fabrication stratégique de l'incertitude sur les ours du Béarn
}

\author{
Laurent Mermet, Farid Benhammou
}

\section{- To cite this version:}

Laurent Mermet, Farid Benhammou. Prolonger l'inaction environnementale dans un monde familier : la fabrication stratégique de l'incertitude sur les ours du Béarn. Écologie \& politique: sciences, culture, société, 2005, 2 ( $\left.\mathrm{N}^{\circ} 31\right)$, pp.121-136. 10.3917/ecopo.031.0121 . hal-02909753

\section{HAL Id: hal-02909753}

https://hal-agroparistech.archives-ouvertes.fr/hal-02909753

Submitted on 31 Jul 2020

HAL is a multi-disciplinary open access archive for the deposit and dissemination of scientific research documents, whether they are published or not. The documents may come from teaching and research institutions in France or abroad, or from public or private research centers.
L'archive ouverte pluridisciplinaire HAL, est destinée au dépôt et à la diffusion de documents scientifiques de niveau recherche, publiés ou non, émanant des établissements d'enseignement et de recherche français ou étrangers, des laboratoires publics ou privés. 
[4] A. Cattan, L. Mermet et al. Identification des facteurs de blocage à ladoption de pratiques agricoles favorables à la réduction des pollutions diffuses - synthèse, AScA - Ministère de l'environnement, Paris, 1994.

[5] J. et L. Schiff, Al/ my children, Pyramid Books, New York, 1972.

[6] L. Mermet et X. Poux, "Recherches et actions publiques à l'interface agriculture-biodiversité : comment déplacer le front du débat? ", Courrier de I'Environnement de I'INRA, $n^{\circ} 41,2000$, p. 43-55; L. Mermet, "Les études d'évaluation entre stratégie et méthodologie. L'exemple des politiques publiques en matière de zones humides ", Gérer et Comprendre, $n^{\circ} 46,1996$, p. 55-64.

[7] Ces quatre dimensions renvoient au modèle de méconnaissance/passivité de J. et A. Schiff, op. cit.

[8] Sur les stratégies antienvironnementales en jeu dans le dossier de l'ours; voir L. Mermet,

“ L'Institution patrimoniale du Haut-Béarn: gestion intégrée de l'environnement ou réaction anti-

environnementale? "

Responsabilité et

Environnement, $\mathrm{n}^{\circ} 21$,

2001, p. 9-21 et

F. Benhammou et

L. Mermet, "Stratégie et géopolitique de l'opposition à la conservation de la nature: le cas de l'ours des Pyrénées ", Natures,

Sciences, Sociétés, vol. 11, $n^{\circ} 4,2003$, p. 381-394. environnementales, de tels dossiers devraient être plus facilement traités. Or il n'en est rien. Leur étude montre à quel point l'action est rendue difficile par des désaccords fondamentaux sur le souhait ou non de rechercher telle qualité environnementale, par des luttes d'appropriation du foncier ou de l'argent public, par des enjeux de pouvoir sur le territoire ou sur les institutions, par des pressions pour favoriser ou bloquer l'expansion de certains circuits économiques.

Et si 1'incertitude n'est pas ici au centre des difficultés d'action, des recherches précédentes sur l'interface agriculture/environnement ont permis de constater que dans ce type de dossiers, elle réapparaît sous un autre angle; nous avons pu constater que le faible niveau de l'adoption de pratiques agricoles favorables à l'environnement allait de pair avec un travail permanent de dénégation des effets environnementaux des pratiques agricoles ${ }^{[4]}$. Pour reprendre les termes utilisés par J. et A. Schiff ${ }^{[5]}$ la « méconnaissance » et la «passivité » (activement préservées) sont deux faces d'un même comportement de non prise en charge des problèmes réels. Ainsi le couple « incertitude/difficulté de l'action collective », n'est-il pas le retournement en négatif du couple «savoir/pouvoir » (belgicisme profond et leitmotiv des études foucaldiennes), une sorte d'assignation d'impuissance à l'encontre du domaine environnemental dans son entier? D'autres recherches sur des thèmes apparentés nous ont livré des constats allant dans le même sens ${ }^{[6]}$.

Résumons ces constats de terrain en une formulation plus générale. Dans de nombreux dossiers d'environnement, des stratégies sont mises en ouvre par des acteurs réticents à la conservation de l'environnement pour maintenir ou pour introduire des incertitudes sur l'existence, la nature, la portée des problèmes environnementaux ou sur les solútions appropriées ${ }^{[7]}$. Volet cognitif des stratégies anti-environnementạles, cette fabrication de l'incertitude contribue à délégitimer les revendications du mouvement social environnemental et les missions des institutions qui en assurent les relais dans l'action publique. En brouillant activement les repères de la discussion sur les objectifs et les moyens des actions elle crée aussi un contexte défavorable au passage à l'action pour traiter effectivement les problèmes environnementaux. C'est ce constat que nous entendons ici développer en deux temps.

Dans un premier temps, nous l'illustrerons par un cas d'école: celui de l'ours du Haut-Béarn. Comme nous l'avons déjà fait pour d'autres thèmes ${ }^{[8]}$, nous nous appuyons sur un cas qui combine des structures et des dynamiques très largement répandues dans les dossiers environnementaux - et en particulier agri-environnementaux - avec une certaine 
facilité d'exposition et de mise en discussion due à la conjonction de plusieurs facteurs: un territoire rèstreint, une relative simplicité du dossier technique, le caractère exacerbé que revêt depuis des années l'enjeu de l'ours, ainsi qu'une tendance à la théâtralisation du jeu politique qui rend manifestes des formes d'(in)action publique et de stratégies géopolitiques qui prennent ailleurs des tournures plus discrètes.

Dans un second temps, nous mettrons en discussion les questions que soulèvent, au regard des thèses de la « démocratie technique », ces stratégies observées de fabrication de l'incertitude. Dans ce cadre, nous déboucherons sur une conclusion contrastée. En effet, en montrant les relations complexes et intimes entre recherche, expertise, débat et action publics, le cas de l'ours illustre leur pertinence. Mais dans le même temps, la manière dont certains acteurs du dossier construisent et exploitent stratégiquement l'incertitude nous invite à la vigilance au regard de discours fréquemment associés à ces approches. Il faudrait, lisonsnous parfois, tourner définitivement la page des « vieilles » analyses qui, sous les vocables de stratégie, d'idéologie, de pouvoir, supposeraient des identités figées, des intérêts rigides, des modes d'actions adversatifs et enfonceraient ainsi dans l'impasse la recherche collective d'une solution aux défis de l'environnement et du développement durable. Il faudrait au contraire, à partir des concepts analytiques de réseau socio-technique, de collectif cosmopolitique, de démocratie dialogique, faire prévaloir des analyses et des dispositifs qui mettent l'accent sur la redéfinition concomitante et négociée des identités, des attentes, des savoirs et des modes d'action. Mais qu'en est-il des rapports de force, des asymétries majeures de pouvoir qui caractérisent la représentation des différentes préoccupations sociales au sein même des dispositifs et des réseaux de savoir? Ne conduisent-ils pas à des processus tels que l'identité, les attentes, les savoirs et les modes d'action des uns - périphériques au sein des réseaux socio-techniques du « rural », comme les acteurs d'environnement ${ }^{[9]}$ - sont activement et constamment remis en cause, grevés d'incertitude? Ne permettentils pas à d'autres - centraux dans le champ, comme les acteurs du secteur agricole réticents à l'environnement - de se renforcer implicitement par la diffusion d'une vulgate localiste ${ }^{[10]}$, par l'hégémonie et la naturalisation de telle ou telle logique technico-économique et des discours d'accompagnement qui la traduisent en termes de politique sociale? Ne débouche-t-on pas sur des cadrages de la recherche qui induisent d'un côté l'accumulation des « savoirs » scientifico-techniques pour le développement et de l'autre celle d' « incertitudes » de plus en plus élaborées sur l'environnement? Telle est bien le questionnement de portée très générale, déterminant aujourd'hui pour le champ de l'en-
[9] Au sens d'acteurs qui cuvrent pour des changements favorables à l'environnement voir L. Mermet et al., " L'analyse stratégique de la gestion environnementale: un cadre théorique pour penser l'efficacité en matière d'environnement ", Natures, Sciences, Sociétés, vol. $13, n^{\circ} 2$, 2005, p. 127-137.

[10] A. Bourdin, La question locale, Paris, PUF, 2000. 
vironnement et du développement durable, que nous voulons aborder ici à partir de 1'exemple des ours du Haut-Béarn.

\section{Combien d'ours exactement? La mal-formulation de questions environnementales, en quête de non-réponses}

Toute analyse approfondie d'un dossier environnemental doit s'appuyer sur une formulation de l'enjeu environnemental dont on considère qu'il fait l'objet d'une responsabilité collective ${ }^{[1]}$. S'agissant de l'ours des Pyrénées, l'enjeu de référence est d'assurer la viabilité à long terme d'une population d'ours bruns vivant en liberté et auto-reproductrice. Nous ne reviendrons pas ici en détail sur l'évolution du traitement de ce dossier depuis « l'alerte » lancée dans les années 1950 , ni sur les multiples rebondissements et conflits dont il a été émaillé $e^{[12]}$. Retenons simplement que l'État a transféré à un syndi-

[11] L. Mermet et al., 2005, op. cit.

[12] Pour nos analyses, voir L. Mermet, Lanalyse stratégique de la gestion environnementale. Illustrée par les tribulations d'un noyau de population relictuelle d'ours bruns (Ursus arctos) dans les Pyrénées Occidentales françaises, document de travail interne RGTE, 1998; L. Mermet, 2001, op. cit. ; L. Mermet, " Homme ou vie sauvage? Société locale ou bureaucratie centrale? Faux dilemmes et vrais rapports de force ", Responsabilité et Environnement, $n^{\circ} 28$, 2002, p. 13-20;

F. Benhammou et

L. Mermet, op. cit. Nous recommandons également l'ouvrage retraçant l'histoire du dossier ours jusqu'en 1993: C. Dendaletche, $\mathrm{La}$ cause de lours, Le Sang de la Terre, Paris, 1993.

[13] À la fois en termes techniques et biologiques, puisqu'il y a aujourd'hui une dizaine d'ours issus de l'introduction dans la chaîne des Pyrénées, et en termes d'image auprès du grand public. cat mixte d'aménagement, 1'Institution patrimoniale du Haut-Béarn (IPHB) une partie importante des financements publics et des responsabilités dans l'instruction des dossiers tant d'aménagement que de protection et que, depuis 1994, le fonctionnement de l'IPHB a conduit au maintien d'une sorte de statu quo, qui voit la population d'ours continuer de s'amenuiser sur le territoire (Haut-Béarn) dont elle assure la gestion, pendant que la quasi-totalité des financements disponibles y sont consacrés à des aménagements pastoraux ou forestiers en montagne. C'est sur ce volet haut-béarnais du dossier de l'ours que portera ici notre analyse. Rappelons simplement qu'il s'inscrit, depuis 1996 dans un cadre plus large. Un deuxième volet du dossier a été ouvert en effet avec l'introduction d'ours « slovènes » dans le département proche de la Haute-Garonne en 1996 et 1997. Ce deuxième volet a connu un réel succès ${ }^{[13]}$. En inscrivant désormais le sort de l'ours des Pyrénées dans un espace plus vaste que le Haut-Béarn, il a privé les responsables de l'IPHB de leur hégémonie sur le dossier de l'ours en France. Le bilan positif de l'opération des Pyrénées centrales met en évidence par contraste l'attentisme et l'inefficacité de l'action de l'IPHB en matière d'ours, sur laquelle se concentrera notre analyse.

\section{Le suivi de l'ours des Pyrénées: un dispositif en réseau}

Avant la fin des années 1970, il n'existait pas de véritables savoirs « scientifiques » sur l'ours dans les Pyrénées. Seuls quelques chasseurs et quelques bergers véhiculaient encore un savoir empirique. En 1954, un médecin et chasseur grenoblois qui avait arpenté les Pyrénées pour 
la chasse à l'ours l'avait compilé dans un premier ouvrage qui soulignait déjà le déclin rapide des populations d'ours ${ }^{[14]}$. Vingt ans plus tard, entre 1976 et 1981, de manière concomitante à l'investissement du dossier de l'ours par le mouvement associatif puis par le ministère chargé de l'environnement, des naturalistes et des biologistes de terrain commencent à s'intéresser de près aux ours pyrénéens. C'est dans ce cadre que s'inscrit en particulier la démarche du jeune biologiste Jean-Jacques Camarra. Originaire des Hautes-Pyrénées, il s'installe en vallée d'Aspe en 1976 dans le but de développer une connaissance fiable des derniers ours. Pour cela, il va à la rencontre de chasseurs locaux, travaille et vit avec des bergers pour récolter leurs savoirs tout en parcourant intensément la montagne. L'objectif est d'ancrer la base de départ de la connaissance de l'ours dans les savoirs locaux montagnards. Mais à l'époque où s'engage ainsi le suivi naturaliste, les chasseurs d'ours qui avaient eu sans doute une connaissance intime de l'animal et de son milieu ont pratiquement disparu. Au bout de quelques temps, J.-J. Camarra constate que les informations recueillies sur l'ours dans les vallées, pour intéressantes qu'elles soient, s'avèrent insuffisantes et souvent peu compatibles avec les constats biologiques de terrain. Il constate aussi la prévalence d'histoires largement mythifiées, ressassées et, au total, d'une connaissance archétypale et simpliste de l'ours, reflet d'une connaissance du milieu montagnard environnant qui a diminué à mesure que les usages de la montagne se modifiaient ${ }^{[15]}$.

Assez rapidement, les résultats de ses propres prospections débordent les connaissances des principaux acteurs: «alors que je présentais des résultats de suivi à des chasseurs locaux, je leur présentais des choses qu'ils ne connaissaient pas et notamment des tanières; ceux-ci m'ont traité de menteur. Les habitants ont toujours l'impression qu'on empiète sur leur jardin secret, alors c'est très délicat ${ }^{[16]} \gg$.

De 1978 à 1983, J.J. Camarra élabore les méthodes nécessaires à l'étude et au suivi des ours. En plus des prospections, il part régulièrement se former en Amérique du Nord où les études scientifiques sur l'ours sont plus avancées. Il travaille de manière indépendante et en synergie avec quelques gardes du parc national des Pyrénées (PNP) et avec le Fonds d'intervention éco-pastoral (FIEP), où G. Caussimont travaille lui aussi à l'étude du problème de l'ours. Ce cadre de collaboration plus ou moins informel devient la base des remontées d'information aux services de l'administration de l'environnement naissante.

En 1981, J.-J. Camara est intégré à l'Office national de la chasse (ONC) et met en place, sur l'initiative de la Fédération départementale des chasseurs, un dispositif de suivi systématique de la population d'ours : le Réseau ours brun. Il forme alors les gardes-chasse de l'ONC,
[14] M. Couturier, L'ours brun, Arthaud, Grenoble, 1954.

[15] G. Di Méo et J. Pradet, "Territoire vécu et contradiction sociales: le cas de la Vallée d'Aspe (Pyrénées Occidentales) ", in G. Di Méo (dir.), Les territoires du quotidien, L'Harmattan, Paris, 1996, p. $49-86$.

[16] J.-J. Camarra, com. pers. 
travaille étroitement avec les agents du PNP, de l'Office national des forêts (ONF) et des bénévoles des associations environnementales. Se met alors en place un système de récolte d'informations sur l'ours, fondé sur la collecte d'indices (traces, poils, crottes, etc.) et sur les témoignages d'observations fournis par les techniciens d'organismes publics, les associations naturalistes et les autres usagers locaux. Dans le courant des années 1980, les études génétiques d'un laboratoire de recherche de Grenoble ont permis de préciser les connaissances concernant les ours. Le suivi et l'identification des individus sont complétés aussi par des dispositifs de photos automatiques.

Dès 1984, après 6 ans d'études de terrain et alors que le dispositif de suivi systématique n'en est encore qu'à ses débuts, on dispose d'études permettant de cerner la situation. L'effectif des ours est connu à quelques unités près. Leur localisation est suffisamment bien connue pour permettre une cartographie précise des zones vitales pour leur reproduction, leur alimentation et leur protection contre le braconnage. Depuis, le suivi n'a cessé de s'étoffer. Jusqu'à ce jour, il a confirmé et précisé les résultats de 1984 et permis de suivre la dégradation de l'état de conservation de la population d'ours (d'une quinzaine au début des années 1980 à cinq en 1995) et d'en cerner les causes avec une précision suffisante pour alimenter la réflexion sur les actions souhaitables et possibles ${ }^{[17]}$.

\section{Première tentative de déstabilisation: dénégation et menaces (1990-1991)}

Pour autant, tout se passe de 1984 à 1990 comme si la connaissance de la situation en matière d'ours ne débouchait sur aucune action suffisamment efficace pour enrayer sa dégradation. Le «plan ours » proposé en 1984 par le ministère de l'environnement s'enlise face à une sorte de résistance passive du secteur agricole. En 1990, la tension monte lorsque le ministère de l'environnement s'engage de plus en plus énergiquement dans la négociation pour de nouvelles mesures. Cet effort échoue devant une radicalisation de l'opposition à la protection de l'ours, orchestrée essentiellement par certains élus, par les milieux socioprofessionnels agricoles et cynégétiques. Cette réaction s'accompagne de tentatives virulentes pour faire table rase des données et analyses disponibles sur l'ours et son milieu, connaissances qui constituent à l'évidence l'un des atouts stratégiques des acteurs favo-

[17] G. Caussimont, Avec le naturaliste, sur les pas de lours brun des Pyrénées, FIEP, Loubatières, Portetsur-Garonne, 1997. rables à de nouvelles mesures. Les experts en matière d'ours - ainsi d'ailleurs que le sous-préfet - vivent alors une période très difficile de prises à partie répétées, allant jusqu'à des menaces de mort. Les acteurs d'environnement sont accusés de mentir délibérément sur le nombre 
d'ours qui serait supérieur à ce qui est présenté. Le tableau est rendu encore plus confus par les rumeurs de lâchers clandestins d'ours qui vont bon train.

La crise se prolonge en un statu quo tendu jusqu'à la création, début 1994, de l'IPHB. Cette institution consacre alors la domination d'un groupe d'élu et de composantes du syndicalisme agricole, au sein du dispositif de concertation qui se retrouve en charge des dossiers concernant l'ours et son milieu, dossiers qui, à l'époque, sont confinés au Haut-Béarn.

\section{Deuxième tentative de déstabilisation: quelle question poser pour ne pas recevoir de réponse? (1995-1996)}

Pendant les tractations sur la création de l'IPHB, l'analyse de la situation et des propositions en matière d'ours a été prudemment mise en attente, renvoyée à un « audit international » qui devait, une fois la situation institutionnelle redéfinie, « mettre à plat » les données du problème. L'audit est lancé en 1995 et est confié par appel d'offre à un groupement constitué d'un bureau d'études spécialisé en environnement, d'un expert américain incontesté en matière d'ours, et de l'un des auteurs de ces lignes. Trois questions sont posées aux experts et c'est à travers leur formulation et le sort réservé aux réponses que vont se jouer pour un temps les tentatives pour ré-expliciter ou pour déstabiliser le diagnostic de la situation.

La première question concerne l'évaluation du dispositif de suivi de l'ours mis en place sous la responsabilité de J.-J. Camarra - celuilà même dont les résultats ont été dénoncés avec virulence par les groupes de pression qui sont maintenant à la tête de l'IPHB. Après examen détaillé des activités, des méthodes et résultats du Réseau ours brun, l'évaluation est sans ambiguïté. «Les informations recueillies sur l'ours des Pyrénées constituent le plus grand ensemble au monde de données obtenues sur une population non marquée (par collier émetteur). [Elles] ont permis d'avoir une compréhension très précise de cette population. Ces données sont suffisantes pour pouvoir prendre des décisions en matière de gestion ${ }^{[18]} \gg$. Cette première réponse met fin brusquement aux tentatives de déstabilisation par dénégation frontale et menaces. Experts et résultats sont réhabilités, revendiqués comme compétence locale et, pendant plusieurs années, il n'y aura plus de contestation sur ce plan. Ce sont les deuxième et troisième questions qui prendront le relais dans la stratégie de fabrication de l'incertitude.

La seconde question concerne le nombre exact d'ours. Or, quelle que soit l'ampleur du dispositif de suivi, il ne permet pas forcément
[18] X. Poux et al., État des lieux de la population ursine et de son habitat dans le Haut-Béarn. Stratégies de conservation et de renforcement éventuel, ASCA - IUCN - Univ. du Montana - JPHB, 1996. 
[19] Les travaux et discussions pour cerner la question cruciale du nombre d'ours qui doivent être présents pour assurer une démographie viable sont évidemment au coeur des recherches et expertises ursines. L'ordre de grandeur qui sert de référence aux discussions depuis le début des années 1980 est un minimum d'une quarantaine d'ours sur le massif, soit une quinzaine dans le Béarn. Pour une référence récente, voir G. Chapron et al., « Which future for the French Pyrenean brown bear (Ursus arctos) population? An approach using stagestructured deterministic and stochastic models ", C.R. Biologies, $n^{\circ} 326,2003$, p. 175-182. d'avoir une réponse absolument exacte. Certes, l'incertitude qui affecte ici le résultat - en l'occurrence, entre 5 et 7 ours - est non pertinente du point de vue de la gestion: le diagnostic de la situation n'est pas à un ours près ${ }^{[19]}$. Pourtant, l'exigence d'un nombre exact est martelée de façon obsédante dans les réunions de l'IPHB. Elle va de paire avec l'affirmation selon laquelle tant que le nombre exact d'ours n'est pas certain, il n'est pas possible d'agir. Cette exigence est selon nous l'une des formes les plus souvent rencontrées que prennent les stratégies de fabrication de l'incertitude. Dans un premier temps, la réponse apportée par l'audit - 5 ours - sera néanmoins acceptée provisoirement, au bénéfice de la réappropriation par les acteurs locaux de l'expertise en matière d'ours.

La troisième question porte sur les évolutions possibles de la situation. La réponse est fournie sous forme de scénarios d'évolution à la fois en matière d'ours et en matière de relation entre ours et développement des vallées. Toute cette partie du rapport est violemment rejetée par l'IPHB, qui met fin à l'audit à mi-parcours et refuse toute forme de mise en discussion, que ce soit sur la teneur des scénarios, sur leur mode d'élaboration ou sur les enseignements que l'on pourrait en tirer. Le président de l'IPHB accuse le groupement d'avoir voulu dicter les solutions. Tout se passe en fait comme si, en ouvrant un débat qui rend plus lisibles les enjeux des décisions à prendre, l'explicitation de scénarios par les experts faisaient peser une pression insupportable sur les décideurs politiques et socioprofessionnels détenteurs du pouvoir au sein de l'IPHB. Ce refus d'expliciter les liens entre choix de développement et conséquences environnementales est à l'évịdence un autre volet majeur des stratégies de fabrication de l'incertitude.

\section{Troisième tentative de déstabilisation: création d'une anti-expertise "valléenne » (2000)}

Suite à l'interruption de l'audit, l'IPHB décide fin 1996 d'une politique présentée comme «équilibrée » : un « pacte d'objectifs » qui prévoit notamment l'introduction de deux ours. Pour des raisons diverses, l'IPHB finit cependant par abandonner ce projet. De plus, les interférences croissantes avec le dossier des ours réintroduits en Pyrénées centrales conduisent à la radicalisation des composantes hostiles à l'ours au sein des groupes de pression qui contrôlent l'IPHB. Dès lors celle-ci, qui depuis 1995 reconnaissait, comme on l'a vu, le travail du Réseau ours brun, va progressivement le désavouer et le critiquer, faisant jouer les ressorts d'une rhétorique de la victimisation et du complot. La lettre d'information de l'IPHB ouvre les hostilités en août 2000 : «d'anciens débats resurgissent; combien y a-t-il réelle- 
ment d'ours? 6 ou plus? Combien y a-t-il effectivement de femelles? Que cache vraiment ce déficit d'information?"

En montant en épingle des dysfonctionnements mineurs et hétérogènes, en accusant frontalement l'État d'être, pour des raisons de pouvoir, au centre d'une entreprise de dissimulation de la réalité de la situation, les responsables de l'IPHB ont semé le doute. Mais ils vont plus loin pour faire tenir de manière durable l'incertitude en proposant leurs propres calculs : il restait 5 ours dont une seule femelle en 1995; or il y a eu 3 naissances depuis $(1995,1998,2000)$; il y aurait donc 8 ours. Certes, 5 et 3 font 8 , mais c'est ici ne pas tenir compte de la mortalité des ours. La mort de l'un des oursons est connue de manière certaine et directe; de plus le dispositif de suivi permet, lorsque l'on ne retrouve pas d'indices pendant longtemps, de faire des hypothèses sérieuses sur la disparition (par décès ou par sortie de la zone) de certains individus.

Les experts du Réseau ours sont alors accusés d'écarter certains témoignages de bergers, qui prouveraient que tous les ours n'ont pas été comptabilisés. L'arrivée sur la scène d'un naturaliste bricoleur, dentiste près de Pau, tombe à point nommé pour alimenter la remise en cause. Celui-ci affirme qu'il sait des choses que Camarra ne veut pas dire ou ne sait pas et, grâce à ses propres appareils de photographie automatiques, il affirme être parvenu à identifier des ours inconnus du Réseau. Certes, lorsque ces clichés ont été discutés de manière approfondie, il lui a bien fallu reconnaître que ces interprétations étaient erronées. Ceci ne l'a pas empêché de communiquer ses résultats, à travers Paris Match notamment, ni d'être reçu plusieurs fois lors de réunions à l'IPHB, où ses prestations sont présentées comme une « contre-expertise » révélant des choses cachées. En mettant en avant ce savoir présenté à la fois comme expert et comme local, I'IPHB remet en cause le système de connaissance de l'ours ${ }^{[20]}$.

Dans un des moments les plus étonnants du dossier de l'ours, les responsables de l'IPHB vont aller plus loin pour s'efforcer de ravaler les résultats de vingt ans de suivi de l'ours au rang d'une expertise « officielle », manipulée et discréditée. Dans un rapport d'auto-évaluation daté de 2001 , les données sur la situation de l'ours sont présentées dans les termes suivants. «Deux points de vue différents sur la vitalité de la population ursine: (1) une analyse technico-scientifique, partagée par quelques valléens, omniprésente au niveau national: la population d'ours s'est au mieux maintenue voire s'est vraisemblablement dégradée; (2) une analyse pragmatique très partagée dans le haut Béarn: "l'homme est lié à l'ours comme l'ours est lié à l'homme" ». En schématisant, « comme l'homme est en train
[20] II est à noter cependant que l'IPHB ne s'en prend pas directement à l'animateur du réseau, J.-J. Camarra. Ses dirigeants ont exprimé à plusieurs reprises le souhait que l'ensemble du suivi passe sous leur responsabilité. 
de revenir dans les vallées, l'ours est en train de revenir aussi ». Les auteurs de l'étude tracent alors deux courbes ${ }^{[21 !}$.

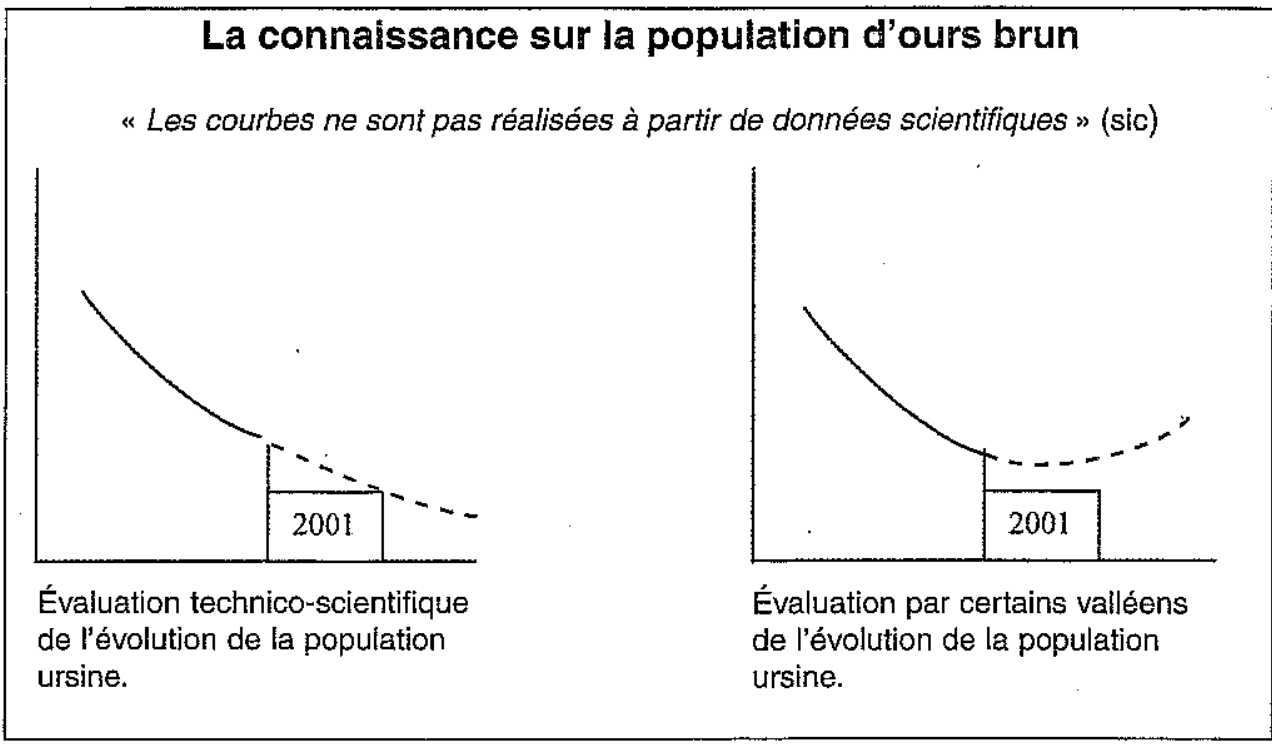

Dans des versions antérieures du texte, « l'innovation » épistémologique était plus étonnante, puisque les auteurs mentionnaient d'un côté un «nombre technico-administratif » d'ours et de l'autre un «nombre patrimonial » d'ours. Il n'en reste pas moins que le fond reste le même: on légitime le fait de mettre sur le même plan toutes les paroles sur la situation biologique, quelle que soit leur origine et leur pertinence. On ré-institue l'ignorance, l'incertitude, en ne donnant aucun poids au travail d'élaboration du dispositif de suivi de l'état de la population ursine, sans pour autant se sentir obligé d'apporter des arguments à l'appui de cette remise en cause. Et le rapport (sur lequel l'IPHB fonde de nouvelles demandes de financement et de délégation de pouvoir pour la gestion de l'ours) conclut sa discussion de la situation biologique de manière impavide: «Ces deux analyses conduisent à des conclusions différentes, sur la connaissance de l'ours, sur le nombre d'individus vivant dans la zone de la charte, sur la vitalité de la population d'ours ».

[21] RCT/A2D, Bilan quantitatif, qualitatif, patrimonial des premiers contrats de programme pluri-annuel 1994-1999 de la charte de développement durable des vallées béarnaises et de protection de l'ours, IPHB, 2001.

[22] E. Rémy et J. Estades, «Sur les traces de l'expert ", Natures, Sciences, Sociétés, vol. 10, $n^{\circ} 2,2002$, p. 31-38.
Dans un tel contexte, où toutes les informations sont mises sur le même plan, les affirmations les moins étayées prennent peu à peu de l'assurance. Elles ne peuvent être qualifiées d'expertise (pas plus que de contre-expertise), faute de méthode de travail et de discussion contradictoire et évaluative, en public, entre experts et reposant au moins en partie sur des échanges écrits. Nous parlerons plutôt d'anti-expertises pour souligner qu'elles visent seulement un effet négatif: la fabrication de l'incertitude par un travail de sape contre le Réseau ours brun dont la crédibilité, la bonne insertion locale, et les résultats peuvent fournir un appui à l'évaluation de l'action en matière d'ours ${ }^{[22]}$. 
En juin 2002, le président de l'IPHB est élu député et Roselyne Bachelot, du même bord politique, devient ministre de l'écologie et du développement durable. Il est temps de franchir encore un pas de plus dans l'institutionnalisation de l'anti-expertise et de l'incertitude. Dans un discours prononcé le 17 février 2003 à l'IPHB, la ministre, se démarquant du travail de ses services et des travaux des experts, indique: "j'ai le sentiment que l'on ne sait pas exactement ce qui se passe, que la question scientifique n'est pas totalement réglée et quand on dit "il y a une population de 5 ou 8 ours" la question n'est pas du tout la même ». Et aux «expertises patrimoniales » de « certains valléens » sur le nombre, la localisation ou la biologie des ours, elle ajoute une « expertise personnelle de la ministre » sur la dynamique de population ursine: «la question n'est pas du tout la même s'il y a une femelle ou s'il y a deux femelles. Dans le premier cas à l'évidence la population est en voie de disparition. Dans le deuxième cas elle est en voie de conservation ${ }^{[23]}$. Donc il faut absolument que cette question soit tranchée. [...] Il faut que ces études soient menées, dans la concertation et dans la cogestion avec vous-même, pour que nous puissions dans le courant de l'année prendre des décisions pertinentes et qui seront acceptées par tous ».

Vingt ans après l'étude qui a permis de bien cerner la situation de l'ours dans le Béarn, dix ans après la dévolution de la gestion à l'IPHB et les expertises qui l'ont accompagnée, on est donc ramené au même point. Les actions déterminantes pour enrayer la disparition de cette population d'ours n'ont pas été menées à bien (dans le Béarn). La même question, sans portée opérationnelle, du nombre exact d'ours est brandie à nouveau comme une incertitude déterminante dont la levée par une procédure nouvelle serait un préalable indispensable qui justifierait, dans l'attente d'une hypothétique réponse unanimement acceptée, l'absence d'accord et l'inaction.

Ainsi est lancée par l'IPHB, en 2004, une opération dite de « constat partagé », pour essayer d'apporter une réponse unanime à des questions dont personne n'est en mesure d'argumenter sérieusement en quoi elles peuvent avoir une portée sur les mesures à prendre.

\section{L'enviromnement, lieu d'imposition scientiste ou zone d'incertitude?}

Pour mettre en discussion les stratégies de fabrication de l'incertitude environnementale que donne à voir le cas de l'ours, repartons du point de vue inverse, qui reproche aux acteurs de la protection
[23] Aucune forme d'argumentation n'est proposée pour étayer cette affirmation, qui n'a fait l'objet d'aucune élaboration technique en amont de son apparition dans le discours politique. 
de la nature de s'appuyer sur des certitudes scientifiques abusivement proclamées, pour couper court au pluralisme politique et à la négociation de la gestion du territoire et de l'environnement. Comme Bruno Latour, qui a poussé fort loin la réflexion sur ce thème $e^{[24]}, l^{\prime}$ exprime brutalement dans un débat oral: «La plupart du temps, l'environnementaliste [...] "connaît" les ours, il a des "faits scientifiques qui prouvent que..."; il ne va pas faire le travail de composition cosmopolitique, mais arriver en disant: "les gars vous faites de l'idéologie, nous, nous avons des faits bien établis" ${ }^{23]} »$.

Une composante d'un discours in humaniste », localiste, anti-scientiste contre la protection de la nature

L'imputation de scientisme ainsi faite aux promoteurs de la protection de la nature se retrouve dans le cadre plus large d'un discours, très répandu aujourd'hui aussi bien dans la sphère acađémique que dans les arènes où se joue la politique environnementale, qui tend à combiner une «vulgate localiste ${ }^{[26]} »$ et une rhétorique « humaniste », qui attribue aux protecteurs de la nature un discours d'exclusion de l'homme et prône par contraste de confier aux « hommes » (comprendre: aux opérateurs du secteur agricole) la gestion des territoires et des ressoúrces.

L'histoire de l'action en faveur de l'ours dans les Pyrénées constitue un contre-exemple particulièrement net à une telle vision. Les premiers chercheurs et experts sur l'ours sont, en 1975, les fondateurs de la principale association de protection de la nature sur le dossier, le Fond d'intervention éco-pastoral, dont le nom et la stratégie font de l'appropriation du problème par les bergers la condition et l'axe stra-

[24] B. Latour, Politiques de la nature. Comment faire entrer les sciences en. démocratie, La Découverte, Paris, 1999.

[25] Séminaire de Cerisy de septembre 2003 sur « Cosmopolitiques et aménagement ».

[26] A. Bourdin, op. cit. [27] On est loin des affirmations récurrentes et sans nuance sur l'idée d'une conservation stricte, excluant l'homme, qui aurait prévalu avant la conférence de Rio en 1992 (voir G. Rossi, L'ingérence écologique, Éditions du CNRS, Paris, 2001). [28] L. Mermet, 2002, op. cit. tégique principal de l'action de conservation de l'ours ${ }^{[27]}$. Nous avons montré ailleurs ${ }^{[28]}$ combien étaient mal fondées les accusations d'antihumanisme et de centralisme si souvent exprimées à l'encontre des partisans de la protection des ours. Quant au Réseau ours brun, on ne peut guère être plus éloigné d'une science « confinée » qui entendrait, loin du terrain et de la société locale, imposer des diktats au nom de ses calculs de principe. Si nous considérons ce dispositif comme un exemple de la « recherche de plein air » prônée par Callon et ses collègues, ce n'est pas seulement à cause de l'opération «Orso » au cours de laquelle chaque printemps plus de cent personnes (agents de l'ONC, de l'ONF, naturalistes locaux, représentants de l'IPHB, etc.) usent leurs chaussures en sillonnant la montagne dans la neige pour relever les indices d'ours de manière synchrone sur toute la chaîne. C'est que par ses membres, le Réseau s'inscrit profondément dans le réseau social et institutionnel de la gestion du territoire; c'est qu'il articule les appar- 
tenances, les formations, les sensibilités locales, régionales, nationales; c'est qu'il traverse l'interface entre la gestion pratique de la faune et la recherche scientifique sur les populations d'ours bruns; c'est que ses procédures de travail le soumettent alternativement aux exigences académiques d'un domaine de recherche international et à celles des gestionnaires du territoire local ; c'est que profanes et spécialistes collaborent au sein du Réseau, s'informent et se forment réciproquement ${ }^{[29]}$. Au demeurant les «traductions ${ }^{[30]} \gg$ associées au travail de ce réseau ne se prêtent guère à d'éventuelles stratégies d'occultation de la connaissance : les indices d'ours (traces, crottes, poils), leurs activités (manger, dormir, se déplacer, lutter pour le territoire), leur démographie (chercher un partenaire, se reproduire, mourir), même si elles demandent une technicité certaine pour être maîtrisées finement, ne se situent pas du tout hors de portée d'une discussion approfondie avec des profanes.

L'exemple de l'ours montre cependant que la construction collective d'un dispositif de «recherche de plein air » n'est pas une condition suffisante pour conduire à une prise en charge efficace d'un problème environnemental comme celui de l'ours. Il y faut aussi (comme le soulignent Callon, Lascoumes et Barthe) des procédures dialogiques appropriées, mais surtout, ajouterons-nous, une situation socio-politique et des stratégies de changement telles que les rapports de force s'articulent de manière opératoire à ce que nous appellerons ici, pour souligner l'homologie, les « rapports de raison ». Les responsables de l'IPHB, dont on a vu les stratégies de fabrication de l'incertitude et qui tendent à répondre « contrat d'honneur » quand on confronte leurs actions à leurs engágements et « confiance » lorsqu'on les interroge sur les faits de la situation, ne semblent guère s'inscrire dans cette perspective.

\section{Que devient l'accusation d'imposition scientiste dans un contexte "de plein air »?}

La dénonciation d'un coup de force scientiste, lorsqu'elle est adressée à des dispositifs ouverts comme dans l'exemple du Réseau ours brun laisse perplexe.

La crainte, exprimée par de nombreux chercheurs, que les dispositifs de suivi et d'expertise environnementale conduisent à une dépolitisation nuisible à la démocratie, paraît salutaire lorsqu'elle s'exerce dans des situations où la déconnexion est trop forte entre débat politique et controverse scientifique ou experte, lorsque l'une et l'autre ne s'informent pas réciproquement. Mais le cas de l'ours appelle notre attention sur la situation inverse: celle où des acteurs du champ politique ou du champ économique agissent pour échapper à l'incidence
[29] Par exemple, la ligne (téléphonique et d'investigation) ouverte à l'intention du public par le Réseau recueille $16 \%$ des informations traitées par le Réseau.

[30] M. Callon, "Éléments pour une sociologie de la traduction. La domestication des coquilles saint-jacques et des marins pêcheurs dans la baie de SaintBrieuc ", L'année Sociologique, $n^{\circ} 36,1986$, p. 169-201. 
que peut avoir sur eux une discussion ouverte et informée de données écologiques. Ils peuvent alors déployer des stratégies pour exercer un contrôle direct de l'institution politique sur l'activité scientifique et les expertises qu'elle alimente ou à défaut s'employer à les discréditer. Le problème n'est plus alors une trop grande distance entre politique et activité scientifique, mais au contraire 1'abolition de cette distance.

Il apparaît alors que l'enjeu de la démocratie technique est de veiller à une juste distance, une juste articulation. C'est ce que de nombreux auteurs ayant travaillé sur l'expertise et la décision environnementale conceptualisent, dans des termes divers. Ainsi, Knoepfel et Descloux distinguent trois espaces de décision («politico-politique », «politicoadministratif et judiciaire » et $«$ politico-scientifique $\left.{ }^{[31]}\right)$ et Philippe Roqueplo met-il en regard «le monde de la science», le «prince » et le «peuple $»^{[32]}$. Au-delà de la diversité des termes, le point crucial est ici que ces instances doivent être à la fois distinctes et articulées. La notion de forum « hybride $e^{[33]}$ » est ici particulièrement appropriée, à condition que l'on file la métaphore jusqu'au bout: la culture d'hybrides ne suppose pas seulement des croisements à chaque génération, mais suppose aussi la conservation dans la durée de lignées parentales distinctes.

L'exemple de l'ours suggère que cette discussion nous conduit bien au cœur du problème. En effet, là où la démocratie technique suppose à la fois autonomie relative et interaction intense entre le jeu politique, le débat scientifique, l'opinion, les médias et les dispositifs dialogiques de concertation, notre matériau de terrain montre à quel point les dirigeants de l'IPHB ont cherché à abolir ces distinctions.

Ici, la dépolitisation ne guette pas sous la forme d'un trop grand éloignement de la science et de l'expertise par rapport au politique, mais au contraire d'un étouffement complet par le pouvoir politique lui-même des effets politiques potentiels de l'investigation sur les données écologiques, techniques, économiques, sociales, de la situation. Cet effort pour dévitaliser la démocratie technique en abolissant les

[31] P. Knoepfel et M. Descloux, "Valeurs limites d'immiscions: choix politiques et déterminations scientifiques ", in J. Theys (dir.), op. cit., p. 255-280. [32] P. Roqueplo, "L'expertise scientifique: convergence ou conflit de rationalités? ", in J. Theys (dir.), op. cit., p. 43-80. [33] M. Callon, 1986, op. cit. spécificités et les autonomies des arènes d'investigation et de discussion se retrouve dans cette boutade du directeur de l'IPHB : « Combien y a-t-il d'ours? 5 selon la police, 8 selon les manifestants ». Le dispositif de recherche en plein air et le pouvoir d'État sont ici amalgamés et assimilé à une instance seulement autoritaire (« la police »). Et par un retournement ambigu, le syndicat mixte, institution politique élue, gestionnaire, détentrice de larges pouvoirs, bénéficiaire et re-distributrice des financements publics, suggère qu'elle s'identifie à la position des victimes et des protestataires (« les manifestants »). 


\section{Conclusion}

\section{D'abord, un épilogue}

Le premier novembre 2004, un groupe de chasseurs se rend dans un vallon béarnais pour $\mathrm{y}$ conduire une battue. La dernière ourse femelle de la souche pyrénéenne y est présente depuis des semaines avec son ourson de l'année. Comme tous les chasseurs de la zone, ils en sont bien informés, grâce au dispositif de suivi. Au terme de la battue, l'ourse aura été tuée. La réaction de l'opinion publique et des acteurs de l'environnement est très vive. La procédure de «constat partagé » n'est pas finie, mais le constat public est clair: c'était bien la dernière femelle parmi les ours de souche. L'avenir de la population est entièrement lié (c'était déjà la conclusion des études du début des années 1990) au renforcement de la population d'ours, auquel l'IPHB décide, fin janvier 2005, de ne pas s'associer. L'incertitude dissipée, la réticence ressort dans sa nudité première. Quinze ans ont passé sans action significative sur le dossier de l'ours dans le Haut-Béarn.

\section{La fabrication de l'incertitude comme stratégie de pouvoir au service de l'inaction environnementale}

Les travaux sur la démocratie technique ont bien montré l'intérêt d'être vigilant face à une possible imposition scientiste de la part des acteurs des dossiers environnementaux, y compris les promoteurs de la protection de la nature. Mais des situations comme celle que nous venons de décrire montrent la nécessité de ne pas laisser cette vigilance dériver vers une accusation automatique de scientisme destinée à disqualifier les porteurs de revendications environnementales. Il importe de se donner des repères pour distinguer les situations où la démocratie technique est menacée par un hégémonisme de la part des scientifiques de celles où c'est un hégémonisme de certaines forces politiques qui la met en péril.

Dès lors que ces repères sont clarifiés - et c'est en réalité un travail complexe, qui engage le positionnement propre des chercheurs dans le débat à la fois scientifique et politique ${ }^{[34]}-$ il devient possible de distinguer entre des attaques contre des certitudes environnementales abusivement affichées et des stratégies de fabrication de l'incertitude qui visent au contraire, comme dans le cas des ours du Béarn, à défaire les réseaux, les maillages, les controverses et régulations qui émergent avec la prise en charge progressive d'un dossier environnemental. L'exemple de l'ours illustre d'ailleurs la diversité des formes que peuvent prendre ces stratégies et le poids dont elles peuvent peser dans le traitement d'un problème écologique.
[34] Le lecteur pourra trouver un développement plus approfondi de ce thème dans $\mathrm{L}$. Mermet, " Ouvrir de nouveaux espaces critiques. Clarifier, renouveler, "pluraliser" les ancrages normatifs des recherches ", in N. Berny, H.R. Billé et al. (dir.), Concertation, décision, environnement. Regards croisés, La Documentažion française, Paris, vol. 4, (à paraître). 
[35] Encore que

l'outrecuidance avec laquelle elles sont affichées et revendiquées comme un modèle pour la gestion démocratique des territoires et de l'environnement nous fasse un devoir de mettre au débat public nos analyses dès lors qu'elles sont fondées sur un réel travail de recherche (investigation, cadrage méthodique, mise en discussion critique). Comme l'écrit Roqueplo (op. cit.): “ En tant que citoyens les scientifiques en particulier dans le domaine de l'environnement - sont investis d'une fonction critique fondamentale : étant ceux qui font métier de savoir, ils sont par le fait même chargés de repérer les faux savoirs". Les fausses incertitudes relèvent à nos yeux du même traitement.

[36] M. Crozier et E. Friedberg, Lacteur et le système, Seuil, Paris, 1977.
En insistant, de manière un peu provocatrice, sur la dimension adversive, agonistique, qui ressort de manière si flagrante de notre terrain, notre propos n'est pas tant d'accentuer la dénonciation de certaines pratiques $^{[35]}$ que de souligner la nécessité d'une prise en considération active, dans les théories de l'environnement, de cette question. C'est l'un des apports d'analyses stratégiques inspirées notamment des travaux de Crozier et Friedberg ${ }^{[36]}$. Ces auteurs soulignent ainsi que «définir un problème, c'est toujours aussi déterminer les incertitudes pertinentes, etc'est donc aussi circonscrire indirectement avec elles la structure de pouvoir du construit humain qui devra le traiter $\gg$. Ce n'est sans doute pas trahir leur pensée que de détourner cette citation de la manière suivante: redéfinir un problème (par exemple en focalisant sur l'exactitude du nombre d'ours) c'est aussi s'autoriser à investir des incertitudes non pertinentes et ainsi circonscrire la structure de pouvoir du construit humain qui pourra ne pas le traiter! On rejoint la relation intime, soulignée par J. et A. Schiff entre « redéfinitions », « méconnaissances » et «passivités ». Bien d'autres vocabulaires conceptuels peuvent ici être mobilisés. L'important est d'aborder clairement la manière dont se nouent ensemble l'incertitude, la structuration des situations de gestion, les enjeux de pouvoir, les stratégies par lesquelles chacun défend ses intérêts, son identité, sa vision du monde.

Outre les constats de terrain et les raisons théoriques qui nous incitent à tenir ensemble les aspects cognitifs et les aspects stratégiques de la gestion environnementale, c'est la situation du champ de l'environnement dans son ensemble qui appelle aujourd'hui, et de manière urgente, cette ré-articulation. Après les blocages des années 1980, après les intenses recompositions des années 1990, le milieu des années 2000 nous confronte à nouveau à une période où se multiplient les blocages, voire les reculs, dans le domaine de l'environnement. La fluidité des apprentissages et des renégociations ne peut pas, à elle seule, fournir les appuis nécessaires pour penser ces nouvelles situations. De plus, les grilles de lectures académiques qui mettent en avant cette fluidité ont déjà été assimilées, récupérées et souvent retournées par les acteurs du jeu politique environnemental eux-mêmes. Dans de nombreux cas - l'ours du Béarn n'en est qu'une illustration - elles fournissent aujourd'hui le vocabulaire et les plis d'un discours qui sert de voile aux stratégies les plus discutables d'imposition technico-économique, d'appropriation du territoire et des financements publics, de rejet des attentes environnementales. 